\title{
Gabapentin improved sensory and motor symptoms in the restless legs syndrome
}

Garcia-Borreguero D, Larrosa O, de la Llave Y, et al. Treatment of restless legs syndrome with gabapentin: a double-blind, cross-over study. Neurology 2002;59:1573-9.

\section{QUESTION: In patients with restless legs syndrome (RLS), is gabapentin more effective} than placebo for improving sensory and motor symptoms?

\section{Design}

13 week randomised (allocation concealed*), blinded (clinicians and patients),* placebo controlled, crossover trial.

\section{Setting}

Madrid, Spain.

\section{Patients}

24 patients who were $33-75$ years of age (mean age 55 y, $67 \%$ women) and were diagnosed with idiopathic RLS (established by the International RLS Study Group). Patients with ferritin concentrations $<20 \mu \mathrm{g} / \mathrm{ml}$ were excluded. 21 patients $(88 \%)$ completed the study.

\section{Intervention}

Patients were allocated to sequence 1 (gabapentin followed by placebo) or sequence 2 (placebo followed by gabapentin). Gabapentin was given at an initial dose of $600 \mathrm{mg} /$ day, up to a maximum dose of $2400 \mathrm{mg} /$ day. The sequences lasted 6 weeks with a 1 week washout period, and then patients crossed over to the alternate sequence.

\section{Main outcome measures}

Difference between groups for change from baseline to week 6 on the RLS Rating Scale (RLS symptom severity classified as mild [0-15 points], moderate [16-25 points], or severe [26-40 points]). Secondary outcome measures included patient global impression of change (PGIC) determined by a mark on a line indicating the change in symptoms from the beginning (lowest score $[0 \mathrm{~cm}]=$ "much worse"; highest score $[10 \mathrm{~cm}]=$ "much improved"), clinical global impression of change (CGIC), sleep quality, and periodic leg movements (PLM). Adverse events were also assessed.

\section{Main results}

Analysis was by intention to treat. Patients who received gabapentin had a greater decrease in RLS Rating Scale score than did patients who received placebo (decrease in mean score $10.5 \sim 2.1, \mathrm{p}<0.001)$. Patients who received gabapentin had higher scores on the PGIC than did patients who received placebo $(83.8 v 63.3 \mathrm{~mm}$, $\mathrm{p}<0.001)$. Gabapentin also improved scores on the CGIC, improved sleep quality, and reduced PLM. Adverse events were more common in the gabapentin group than in the placebo group $(48 \% v 21 \%, \mathrm{p}<0.05)$.

\section{Conclusion}

In patients with restless legs syndrome, gabapentin was more effective than placebo for reducing sensory and motor symptoms but had adverse effects.

*See glossary.

\section{COMMENTARY}

RLS is currently diagnosed clinically, because no confirmatory laboratory test is available, and is treated with such drugs as levodopa, dopamine agonists, and opioids. Practice parameters of the American Academy of Sleep Medicine favour dopaminergic agents for the treatment of RLS. ${ }^{1}$ The choice of outcome measures used in clinical trials in RLS is very important. Although validated rating scales have been used in some studies, ${ }^{2}$ the study by Garcia et al applied a new rating scale that is subjective and not yet validated.

RLS is a lifelong condition, whose symptoms may gradually worsen with age. To assess clinically relevant outcomes of new drug treatments, their short term efficacy must be unequivocally affirmed before doing long term evaluations.

Although this study provides support for a possible new treatment, it does not provide sufficient evidence about the efficacy of gabapentin for RLS. A particular concern is the risk for a carryover effect, which was not considered in this study. The 1 week washout period between gabapentin and placebo was probably calibrated on the half life of gabapentin (5-7 h), although no evidence exists that its clinical action is short lasting. Blinding of the treating physician is another concern because gabapentin was increased during the study based on therapeutic efficacy. Inadequate blinding may have influenced the outcome by the use of subjective elements in the rating scales. Furthermore, the results for gabapentin and placebo were presented separately, whereas in a crossover design, it is better to report within individual comparison of treatments (paired analysis). The safety of gabapentin remains unclear, because the incidence of adverse events from gabapentin was relatively substantial (48\%). Based on the available information, gabapentin should not be considered a first choice treatment for RLS.

Alberto Albanese, MD Graziella Filippini, MD Istituto Nazionale Neurologico Carlo Besta Milan, Italy

1 Chesson AL Jr, Wise M, Davila D, et al. Practice parameters for the treatment of restless legs syndrome and periodic limb movement disorder. An American Academy of Sleep Medicine Report. Standards of Practice Committee of the A

2 Krishnan PR, Bhatia M, Behari M. Restless legs syndrome in Krishnan PR, Bhatia M, Behari M. Restless legs syndrome in 2003;18:181-5. 